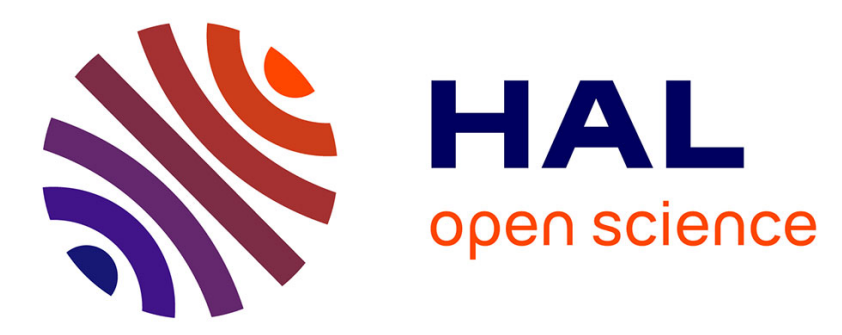

\title{
Neural learning of the topographic tactile sensory information of an artificial skin through a self-organizing map
}

Ganna Pugach, Alexandre Pitti, Philippe Gaussier

\section{- To cite this version:}

Ganna Pugach, Alexandre Pitti, Philippe Gaussier. Neural learning of the topographic tactile sensory information of an artificial skin through a self-organizing map. Advanced Robotics, 2015, Special Issue: Robotic Sensor Skins 29 (21), pp.1393-1409. 10.1080/01691864.2015.1092395 . hal-01254924

\section{HAL Id: hal-01254924 \\ https://hal.science/hal-01254924}

Submitted on 12 Jan 2016

HAL is a multi-disciplinary open access archive for the deposit and dissemination of scientific research documents, whether they are published or not. The documents may come from teaching and research institutions in France or abroad, or from public or private research centers.
L'archive ouverte pluridisciplinaire HAL, est destinée au dépôt et à la diffusion de documents scientifiques de niveau recherche, publiés ou non, émanant des établissements d'enseignement et de recherche français ou étrangers, des laboratoires publics ou privés. 


\title{
Neural Learning of the Topographic Tactile Sensory Information of an Artificial Skin through a Self-Organizing Map
}

\author{
Ganna Pugach ${ }^{a, b} *$, Alexandre $\operatorname{Pitti}^{a}$ and Philippe Gaussier ${ }^{a}$ \\ ${ }^{a}$ ETIS, UMR 8051 / ENSEA, University of Cergy-Pontoise, CNRS, F-95000, Cergy-Pontoise, France; \\ ${ }^{b}$ Energy and Metallurgy Department, National Technical University of Donetsk, Krasnoarmeysk, \\ Ukraine.
}

\begin{abstract}
The sense of touch is considered as an essential feature for robots in order to improve the quality of their physical and social interactions. For instance, tactile devices have to be fast enough to interact in real-time, robust against noise to process rough sensory information as well as adaptive to represent the structure and topography of a tactile sensor itself - i.e., the shape of the sensor surface and its dynamic resolution. In this paper, we conduct experiments with a self-organizing map (SOM) neural network that adapts to the structure of a tactile sheet and spatial resolution of the input tactile device; this adaptation is faster and more robust against noise than image reconstruction techniques based on Electrical Impedance Tomography (EIT). Other advantages of this bio-inspired reconstruction algorithm are its simple mathematical formulation and the ability to self-calibrate its topographical organization without any a priori information about the input dynamics. Our results show that the spatial patterns of simple and multiple contact points can be acquired and localized with enough speed and precision for pattern recognition tasks during physical contact.
\end{abstract}

Keywords: tactile sensor; artificial skin; piezo-resistive material; Electrical Impedance Tomography; neural networks; self-organizing maps.

\section{Introduction}

One great function of the brain is its ability to adapt dynamically to any structural changes observed in the incoming sensory signals. For instance, considering tactile perception, the brain represents the body in a plastic manner, constantly readjusting the body image based on its incoming sensory information: e.g., in situations of abrupt changes occurring during tactile illusions or when one limb is missing or when the brain is injured, it has been observed the more or less rapid re-adaptation of the somatosensory neurons to the new spatial configuration of the body [1-3]. These results show the importance of learning within the lifespan.

Having robots capable of developing such tactile capabilities with the extra possibility of an adaptive body image would provide them with human-like features of self-calibration (by learning the physical extents of the body), of robustness and adaptability (when dealing with unexpected changes in tasks or in the environment). For instance, many robotic researchers use tactile devices to calibrate the body physical limits $[4,5]$ in order to detect the contact area when grasping and manipulating objects [6-8], or to estimate the robot spatial location in order to avoid self-contact or collisions with the environment, or to induce the compliance control based on the robot external tactile feedback [9]. But in these tasks, the tactile perception is tailored to the robotic device and it is difficult to make its structure plastic enough with respect to the changes in the task or in the body. In tactile devices, the information is processed mainly by

*Corresponding author. Email: ganna.pugach@ensea.fr 
means of static parametrical models which are configured once during the initialization setup and which require the computation of nonlinear equations for every time cycle. Despite these problems, they are often more accurate in terms of precision than machine learning algorithms, although they are weaker estimators in presence of external noise or external perturbations and changes (e.g., modification of the sensor properties). In comparison, machine learning algorithms are capable of overcoming the problem of changes in sensory inputs when the body evolves in time. We can cite the work of Bongard and colleagues, for example, who use the evolutionary algorithms to adapt the motor control to the new body image [10].

In the context of haptic devices, various types of sensors have been proposed to transform tactile information into electrical signals [11-13], but not all of them are good for dynamic adaptation when the structure of the sensory information changes. Most of the proposed techniques use an array of isolated unitary devices, piezo-resistive or piezo-capacitive, that covers a surface with small receptive fields [14-16]. The advantage of using grids is to have a look-up table of isolated tactile points with the same precision for each unit that can be mounted quite easily on a specified surface but in order to have an appreciable spatial resolution, it is necessary to cover the surface with very small devices, like MEMS [17]. One practical disadvantage is that as the number of units grows within the structure, the design of the electronic circuits becomes more complex. This implies that it is necessary to connect the units with a large number of wires and also to multiplex them, which can result to bigger power consumption and to possible heat problems that can perturb the device measurement accuracy.

The opposite strategy is to consider a uniform material whose physical properties can be used to process the information; a design principle known as a morphological or physical computation [18]. This material can be seen as a tactile sheet similar to an artificial skin that can transform a density distribution of pressure forces into an ohmic density distribution in the electrical field. The advantage of the second method over the former is that it involves covering large areas of a robot surface various in shape with a flexible and stretchable material similar to the human skin fitting the curves of the robot surfaces with a good ratio between the electronics mounted and the surface size. Its main disadvantages come from (i) its processing time using classical methods which require computing the whole electrical field on a robot surface with the help of parametric equations, which can be quite time consuming, and (ii) the dynamic resolution of the sensor, which may have a poor resolution in comparison to unitary devices.

Although taxel-based sensors can distribute the contact over many taxels and have large receptive fields due to a compliant skin that they are covered, tactile sheets can instead balance the difficult tradeoff between surface/precision/cost/speed in comparison to unit-based devices. Thus, we can categorize devices in three cases: taxel-based sensors with narrow receptive field that use no morphological computation; taxel-based sensors with wider receptive fields, which use morphological computation combined with the grid layout [19]; and tactile sensors where the sensing is due purely to deformation of the sensor surface.

Despite the drawbacks of tactile sheets in comparison with single tactile units, we propose to use the advantages of machine learning techniques to tackle their recurrent problems. We propose to model in robots touch perception similar to humans by means of a tactile surface like an artificial skin from which we build up an artificial neural network (ANN). ANNs are capable of learning in a self-organized manner the electrical activity on the tactile surface in real time by adapting (1) their neural organization to the spatial topology of the sensory inputs and (2) their neural activity to the precision range of the sensors. Although some of these properties can be retrieved by classical parametrical methods, ANNs are more adaptable to learning the device topology and can compute tactile outputs more rapidly and sometimes more precise. Some ANNs (e.g., like self-organizing maps) can even follow the topographic organization of sensory areas with a higher spatial resolution to represent finely some receptive fields [20]. Machine Learning algorithms have been used in grid-like devices with perceptrons and support vector machines have been employed in surface-based tactile devices [21]. But to our knowledge, it is the first time that neural networks are employed in surface-based tactile devices for learning 
the spatial topography. Meanwhile, there have been some studies using SOMs with taxel-based sensors $[22,23]$ but only to categorize objects or types of interactive touch, not the spatial topography of the sensory device and the weight information of object on it.

In our experiments, we compare the neural network method with classical parametric solving methods and we show that neural networks provide greater robustness and greater adaptability to spatial localization as well as faster responses to estimate the resistance distribution on the tissue surface. In case of surfaces of different shapes - square, circular or asymmetric - self-organizing maps can learn easily the spatial topology of the tactile device. Once the topology learned, the neurons compute the relation between the pressure sensitivity and the spatial location at the contact point, which permits to detect multi-touches activity and also discriminate one object shape on its surface (a triangle, a square or a circle).

The paper is organized as follows. The second section describes the generalized parametric method known as Electrical Impedance Tomography (EIT) to estimate the pressure density distribution on the material surface. The third section describes the pressure sensitivity and spatial resolution of the material. The fourth section explains our approach based on neural networks (self-organizing maps) to learn the pressure density distribution. Section five represents the experimental setup and findings (1) in learning different tactile surface shapes: a square, a circle and (2) in detecting objects with different geometrical shapes on the surface: a cube, a cylinder, a prism and an oblong object and (3) in estimating the weights and quantity of objects on the surface: up to 3 objects from $20 \mathrm{~g}$ up to $100 \mathrm{~g}$. We show that self-organizing maps are able to replicate the properties of classical imaging reconstruction methods and go beyond. Finally, in the sixth section, we draw the conclusion and outline our future investigations.

\section{Electrical Impedance Tomography}

In order to estimate the conductivity and permittivity distribution in an electrically conductive material, one popular technique is the electrical impedance tomography (EIT). This non-invasive technique is particularly used in medical imagery to measure the voltage change on the skin surface around the chest with a small current applied at the electrodes. EIT aims at reconstructing a tomographic image of the conductivity distribution within an object from electrical stimulations by injecting currents and measurements at electrodes placed on the surface of the investigated object. The advantage of this technique over more traditional imaging modalities (CT, MRI, PET, ECT) is that it is considered safer by merits of the small currents injected and more affordable by merits of the simplicity of its design, which can be constructed at low-cost and in a very portable size $[24,25]$.

If the material's conductivity has the property to vary locally, the EIT method can be used to sense touch pression and its spatial location. Kato et al. [26], Yao et Soleimani [27] realized a fabric sensors and Nagakubo et al. [28] and Alirezaei et al. [29, 30], Tawil et al. [31] realized a skin-like device for robots based on this principle: by injecting currents and measuring voltages from electrodes connected on the borders of a conductive fabric, they reconstructed the local resistivity changes response from any pressure applied to the material. Nagakubo and Alirezaei concentrated on the development the flexibility and stretch tactile sensor for an integration in robotics as an artificial skin which can be implemented over complex 3D surfaces and also highly stretching areas. Tawil et al. [31] placed emphasis on classification the modality of different types of touch on an experimental EIT-based skin for human-robot interaction.

\section{$2.1 \quad$ EIT acquisition using the neighboring method}

One popular technique for EIT reconstruction is the neighboring method [32]. Its principle is as follows: an electrical current is injected to neighboring electrodes and voltage is measured from other pairs of electrodes. Once all the pairs have been scanned, it is possible to estimate the 


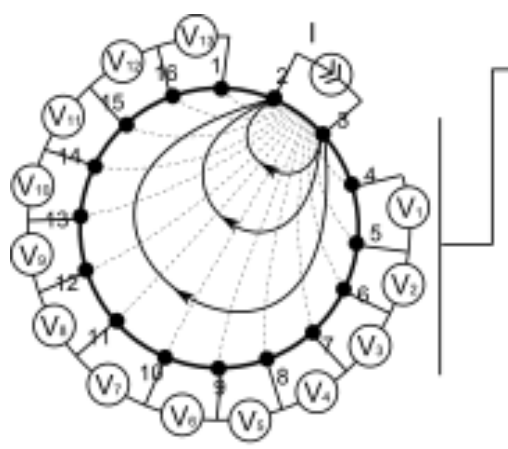

step 1

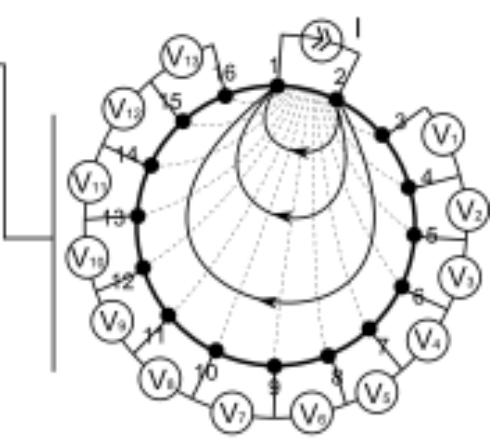

step 2

Figure 1. Neighboring method of data collection for EIT showed for a volume conductor and 16 spaced symmetrically electrodes. The voltage is measured 13 times for 16 different injection current, which correspod to the input matrix $U$ of $13 \times 16=208$ elements.

impedance between the equipotential lines by inverse methods.

We explain the idea of this method for a cylindrical volume conductor with 16 electrodes placed symmetrically on its surface as displayed in Figure 1. The first step is to inject the current between electrodes 1 and 2, which induces an increase of the current density in this particular portion of the material; in other portions, the current density decreases rapidly with respect to the distance to the two electrodes.

To estimate the impedance between the equipotential lines that intersect the two electrodes, the voltage difference is measured 13 times between the other pairs of electrodes (i.e., between electrodes $3-4$, then $4-5$, then $5-6$, etc...). Once the cycle is finished, the next step is to inject the current from the next pair of electrodes $2-3$ and to measure again the 13 potential differences till the end of the sequence, which means 16 times for the current injected between electrodes $4-5,5-6,6-7$ and etc... We obtain then the voltage matrix of dimension $13 \times 16=208$ cells corresponding to 13 measurements of the voltage drop for each current injection between the 16 pairs.

\subsection{Inverse model for EIT reconstruction}

Once the voltage matrix is obtained, we can compute the resistance distribution with the Newton's method for example. This method minimizes the error between the potential difference $V_{i}$ obtained by forward modeling and the $V_{m}$ measured from the conductive sensor. The forward model $(\nabla \cdot \sigma \nabla \phi=0)$ is obtained from the Maxwell's equation where $\sigma$ is the conductivity distribution, $\phi$ is the electrical potential and from a finite element method (FEM) model of the sensors body (see [33] for more details):

$$
V_{i}=F\left(\gamma_{i}, q\right)
$$

where $\gamma_{i}$ is the actual resistance distribution and $q$ is the current stimulation pattern.

The effect on the boundary voltages for a small change in the resistivity of the FEM elements is represented by a Jacobian matrix $J_{i}$ from which we can calculate the actual resistance distribution $\gamma_{i}$. An iterative process, starting from an initial resistance distribution, converges towards the real resistance distribution. Equations for one step of the method correspond to:

$$
\min \left\{\left\|V_{m}-V_{i}\right\|^{2}+\alpha^{2}\left\|R \gamma_{i}\right\|^{2}\right\}
$$


where

$$
\gamma_{i+1}=\gamma_{i}+\delta \gamma_{i}
$$

and

$$
\delta \gamma_{i}=\left(J_{i}^{T} J_{i}+\alpha^{2} R^{T} R\right)^{-1} J_{i}^{T}\left(V_{m}-V_{i}\right) .
$$

The term $J_{i}=\frac{\partial V_{i}}{\partial \gamma_{i}}$ is the gradient of the forward model, with respect to the resistance distribution. The introduced term $\alpha^{2}\|R \gamma\|^{2}$ is a regularization term to stabilize the response. The parameter $\alpha$ describes the regularity of the calculated resistance distribution. The hyper-parameter $\alpha$ describes the smoothness of the calculated resistance distribution and $R$ is a regularization matrix. We have used the Laplace type prior for $R$ [34], but many other prior matrices could also be used. The regularization parameter $\alpha$ has been selected empirically to be $10^{-3}$ as the, providing the best possible result.

The mathematical aspect of the EIT reconstruction is an ill-posed non-linear inverse problem. The main drawback is that the reconstructed image is not necessarily a unique and stable solution, but the small changes in the data (e.g. electrical noise) cause large changes or ruin the entire estimation results. This ill-posed problem can to be solved by regularization [35]. In section 4 we propose to analyze a neural method for solving the inverse model and how it can help to avoid the problem described above.

\section{Material properties of the tactile polymer}

\subsection{Piezo-resistive sensitivity}

The piezo-resistive material used in our experiments is the Velostat film (3M). It is made of opaque, volume-conductive, carbon-impregnated polyolefin whose resistance decreases when pressured; its volume resistivity is around $500 \Omega \cdot \mathrm{cm}$. Figure 2 shows the response of a small rectangular conductive layer of dimension $80 \times 35 \mathrm{~mm}$ connected to 4 electrodes, with one pair for current injection and the other for voltage measurement. The experiments were performed with conductive objects and non-conductive objects in Figure 2 (a) and (b) for the same weight $100 \mathrm{~g}$ applied. Moreover, the surface of the contact area is circular with a diameter of $25 \mathrm{~mm}$.

Following data collection for different values of the weight (i.e. $100 \mathrm{~g}, 300 \mathrm{~g}, 500 \mathrm{~g}, 800 \mathrm{~g}$ ), Figure 3 shows the relation between the pressure on the sensor surface and the resistance variation. We established this relation for both conductive and non-conductive objects. The contact with the conductive objects provokes a significant variation of the resistance $\Delta R_{\text {cond }}$ in comparison to initial value (i.e. from $1.2 \mathrm{k} \Omega$ to $4 \mathrm{k} \Omega$ for the chosen pressure range). The non-conductive objects induce a lower variation of the resistance $\Delta R_{\text {non-cond }}$ to the sensor surface (i.e. from $50 \Omega$ to $150 \Omega$ for the chosen pressure range).

The conductive material varies weakly its resistance value in case of non-conductive objects, but the type of change of resistance is the same as when you press with the conductive objects. Its resistance changes slighty in reaction to different pressure values and no clear discrimination is possible. Therefore, we can assume that the Velostat film works in a quasi-linear regime with the capability to discriminate the pressure applied by conductive objects. For the case of nonconductive objects, the conductive polymer possesses a high hysteresis which does not make it the most suitable material to use since its resistance does not return correctly to its initial value after pressing on it [36].

Finally, one conductive layer (for example conductive aluminum laminated fabric) was added between a nonconductive object and rubber, and the same type of the characteristics (Figure 3) as in the interaction with a conductive object was obtained. 

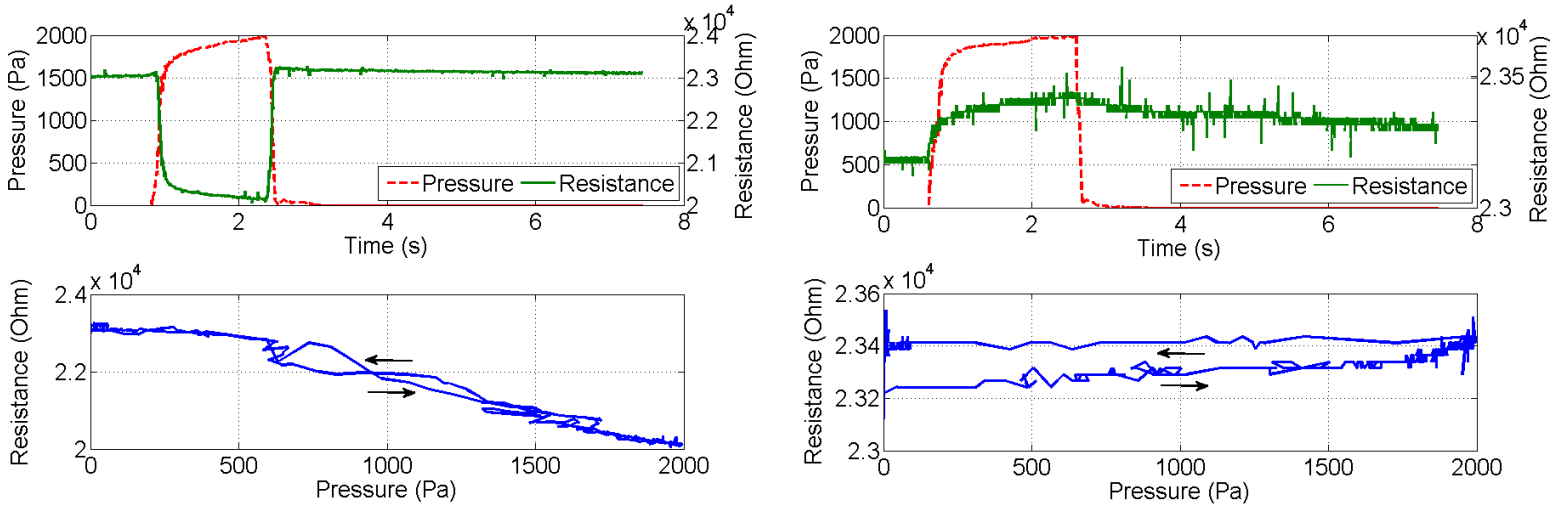

(a) conductive weight

(b) non-conductive weight

Figure 2. Comparison of the piezo-resistive properties of the conductive material to conductive and non-conductive objects; resp. (a) and (b) for the same weight $100 \mathrm{~g}$. On the top chart, the pressure value in $\mathrm{Pa}$ at the contact point and the ohmic value of the resistive material. On the bottom chart: Phase plot of the dependence of the material resistance on the pressure. The conductive pressure point shows no hysteresis with respect to the non-conductive pressure point. The ohmic variations also reveal more amplitude for the conductive pressure point (several hundreds of ohms) than the non-conductive one (several dozens of ohms).

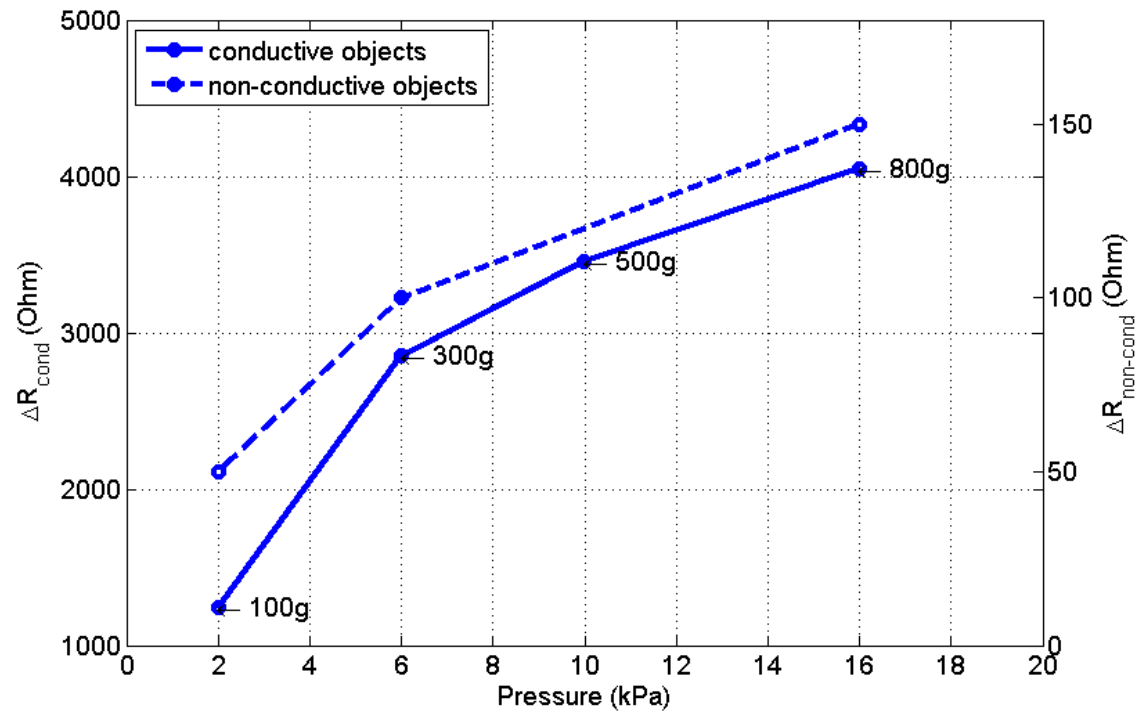

Figure 3. Comparison of the variational resistance $\Delta R$ of the conductive material in its contact point for conductive and non-conductive objects of various weights; resp. the continuous and dashed lines. The results are similar to those in Figure 2, so the fabric is more sensitive to conductive objects put on its surface than non-conductive ones for which the ohmic variation $\Delta R$ is smaller but still can be detected.

\subsubsection{Pressure estimation vs spatial distance to electrodes}

Our previous experiment was conducted on a small rectangular conductive fabric $(80 \times 35 \mathrm{~mm})$ to measure the relationship between the surface resistance of the conductive fabric and the pressure at the contact point. The purpose of the current experiment is to measure the variation in resistance of the conductive fabric with respect to spatial positions. We conducted our experiment on a larger surface, a disc of diameter $200 \mathrm{~mm}$. Sixteen electrodes were placed uniformly on its circumference and a constant DC current $(200 \mu \mathrm{A})$ was iteratively injected into electrode pairs while potential differences were measured for each current injection. The chosen measurement strategy is again the EIT's neighboring method and due to the larger surface of the conductive fabric, the current flow is lower in the central region than in the periphery. 


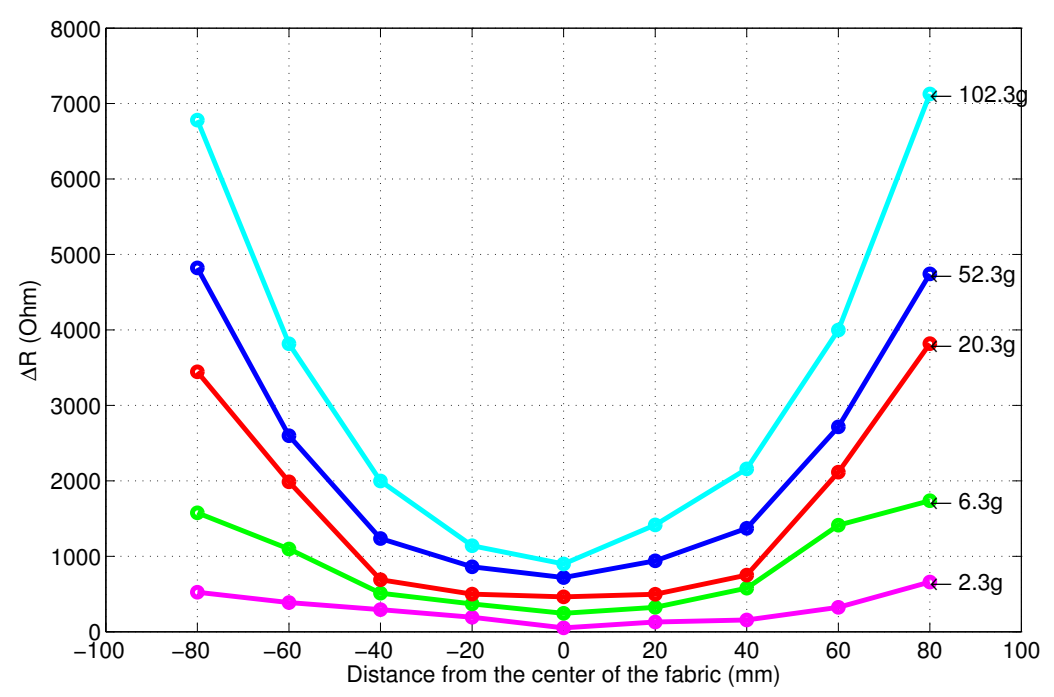

Figure 4. Comparison of the variational resistance $\Delta R$ of the conductive material with respect to spatial distance from the center for conductive and non-conductive objects of various weights. For large surface above $10 \mathrm{~cm}$ radius, there is a nonlinear relationship between the object position on the tactile sensor, its actual weight and the variational resistance $\Delta R$ of the conductive material, which give an overall imprecision up to several centimeters in the location of the contact point on the tactile surface.

To facilitate visualization, several measurement were taken for five weight values $(2.3 \mathrm{~g}, 6.3 \mathrm{~g}$, $20.3 \mathrm{~g}, 52.3 \mathrm{~g}$ and $102.3 \mathrm{~g}$ ) which all had the same circular contact area of diameter $16,25 \mathrm{~mm}$ in order not to influence the electrical field. The change in resistance was measured in 9 positions spaced uniformly by $20 \mathrm{~mm}$. All the measurements started from the same initial position, the disc center.

Figure 4 shows the variation of resistance as a function of spatial position for five weight values. Each variation of the tactile resistance value $\Delta R$ is averaged from 208 values measured. Indeed, for each current injection, 13 measures of electrical potential between pairs of electrodes are made and so for a total of 16 current injections $(13 \times 16=208$ measurements $)$. The observed resistance changes $\Delta R$ have a convex parabolic profile for each weight value. In addition, the variation of the resistance is greater in the vicinity of the electrodes at the center of the fabric. For example, for the weight of $102.3 \mathrm{~g}$, the amplitude variation of the fabric resistance at its boundary is about seven times higher than at the center. The further away we are from the center, the higher the fabric sensitivity increases because the flow of the injected current is higher in the periphery than in the center. The fabric sensitivity in the center can be improved by placing an electrode in the center as proposed in the works [37, 38]. A problem arises from these observations: for different weights, the change in resistance can be the same depending on the position of the contact point. Thus, it may be non-trivial to find the value of a weight from the single measurement of $\Delta R$.

\subsubsection{Spatial resolution}

We assessed the sensitivity of the touch sensor for different spatial positions in the previous experiment. This time, we study the spatial resolution of artificial skin under the same experimental conditions. The purpose is to check experimentally how precise is the detection of ohmic variation with respect to a displacement of the order of only few millimeters. For this purpose, a weight of $102.3 \mathrm{~g}$ is used which is moved back and forth from the center to the perimeter with a displacement about $2.5 \mathrm{~mm}$ per measurement. In comparison to the results obtained in Figure 4, it is seen that the sensitivity varies depending on the position of the contact point. In order to facilitate the visualization of $\Delta R$, the weight is placed on the fabric before data acquisition, thus establishing the reference value. Then, the weight is moved manually twice by $2.5 \mathrm{~mm}$.

Figure 5 (a) and (b) show the results of moving the weight in both directions. In these plots, 
the peak variations indicate the transitional phases when we move the weight. In Figure 5 (a), the ohmic change between two positions, the distance between which is $2.5 \mathrm{~mm}$, is of the order of a hundred ohms and the artificial skin finely detects this change.
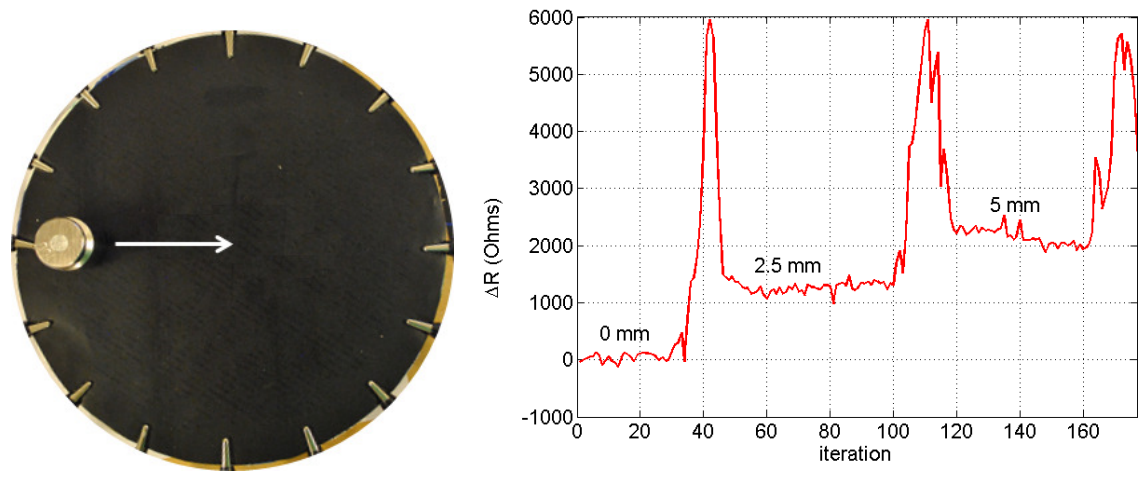

(a)
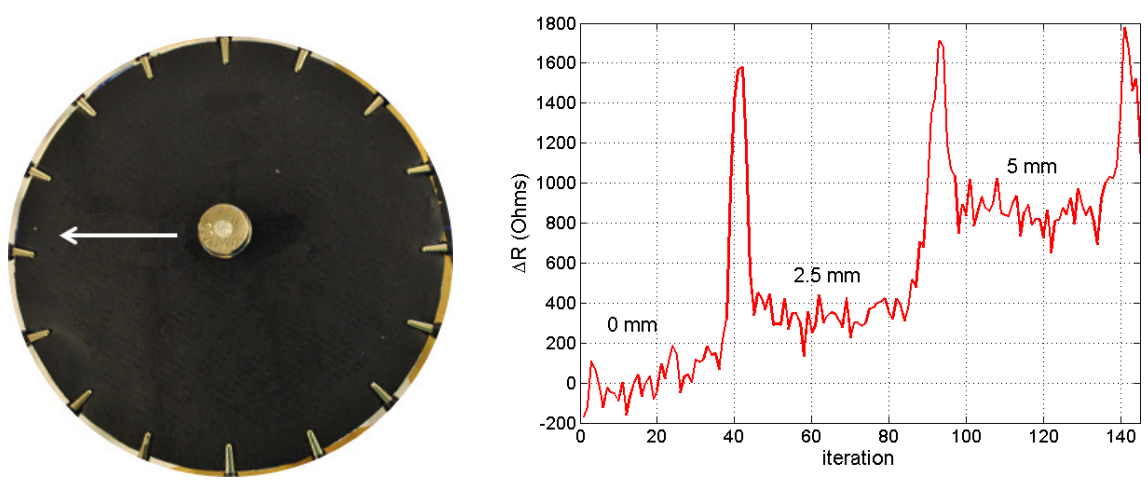

(b)

Figure 5. Time series of the ohmic variation $\Delta R$ of the conductive material with respect to spatial displacement (in mm) of a weight of $100 \mathrm{~g}$ forward and backward; resp. (a) and (b).

Similarly, Figure 5 (b) shows ohmic variations of the order of several tens of ohms when the displacement is from the center of the circular tactile fabric to the edge. The results of these experiments indicate that a $2.5 \mathrm{~mm}$ displacement is detected by the tactile sensor regardless of the $102.3 \mathrm{~g}$ weight position and there is no hysteresis effect.

\subsubsection{Pressure resolution}

Pressure resolution corresponds to the lowest weight which can be detected by the artificial skin. Figure 4 shows the variation of resistance for a displacement of five different weights along the sensor surface. For the lightest weight (2.3g, which corresponds to 1 cent euro coin), we can observe a non-zero $\Delta R$, especially at the center of the touch sensor $(\Delta \mathrm{R} \approx 51.6 \Omega)$, which is the least sensitive area indicated by the value 0 in abscissa. To extend these observations, we plot the ohmic variations at the center of the artificial skin for five different weights, see Figure 6 . As shown in the figure, the profile of the curves is logarithmic but still linearly proportional with respect to the weights. 


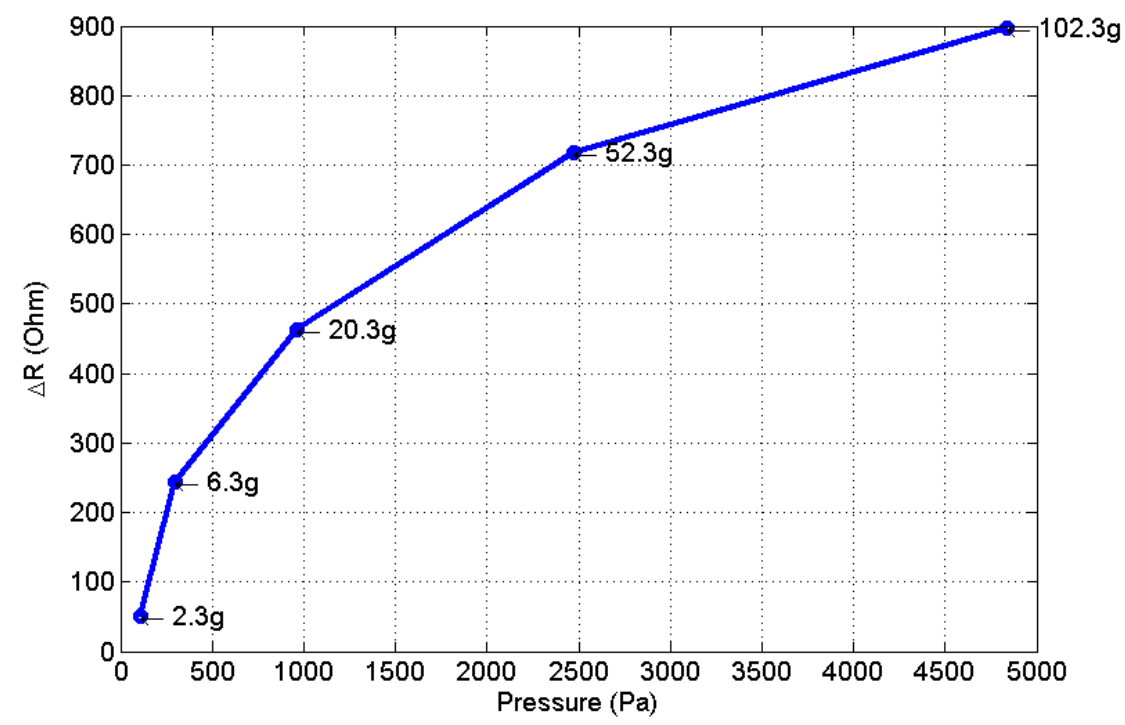

Figure 6. Relation of the variational resistance $\Delta R$ of the conductive material with respect to Pressure $(P a)$ and the weight of objects (same contact point).

\section{Proposed method}

\subsection{State of art in neural network technique for image reconstruction}

Artificial neural networks are well-known optimization methods, highly parallel and distributed with a significant level of learning capability. They have several advantages over classical signal processing methods such as nonlinear regression, rapid adaptability to the input space, fault tolerance to noisy inputs. In previous research, different types of NN were used to solve the inverse problem in EIT such as Bayesian neural networks [39] or Radial Basis Function Neural Networks (RBFNN) [40-42]. Adler and Guardo [43] presented a reconstruction algorithm using neural network techniques, which computed a linear approximation of the inverse problem directly from finite element simulations of the forward problem. NN adapted to the geometry of the medium and to the signal-to-noise ratio (SNR) used during network training.

Table 1 compiles the different tasks where NN were used for image reconstruction on the basis of the EIT method.

\subsection{Self-Organizing Maps}

Self-organizing maps (SOMs), especially Kohonen maps, are a powerful mechanism for analyzing and visualizing multidimensional data $[54,55]$. SOMs are an unsupervised learning method that is applied successfully to various tasks. In image processing, for example, SOMs are used for image compression, feature extraction, segmentation (pixel-based or feature-based), object recognition (pixel-based, feature-based) as well as image understanding [56]. In comparison to the supervised neural networks presented in the previous section, SOMs are a model-free technique, which does not require knowledge about the structure of the input space (e.g., a finite element model of the tactile surface). Another advantage is that they are also bio-inspired methods mimicking the topographical organization of the brain to represent the sensory signals $[2,57]$. One disadvantage is that they can produce a generalization error higher than for the model-based techniques if the input database is not well defined. 
Table 1. A review of Neural Network approach to image reconstruction in EIT

\begin{tabular}{|c|c|c|c|}
\hline Explorers & $\begin{array}{c}\text { Technic \| Electrodes \| } \\
\text { Methods }\end{array}$ & Detection success & NN || Learning \\
\hline $\begin{array}{l}\text { Pandey and Clausen } \\
(2011)[42]\end{array}$ & $\begin{array}{l}\text { EIT \| } 16 \| \\
\text { opposite method }\end{array}$ & $1.5 \%$ limit errors & $\begin{array}{l}\text { RBFNN is constructed and trained by } \\
\text { orthogonal least square (OLS) algorithm }\end{array}$ \\
\hline $\begin{array}{l}\text { Nejatali and Ciric } \\
(1997)[44]\end{array}$ & EIT \| 16 & $2.6 \%-14.7 \%$ & $\begin{array}{l}\text { BPNN (back-propagation) and Forward } \\
\text { problem solving module }\end{array}$ \\
\hline $\begin{array}{l}\text { Stasiak et al. } \\
(2007)[45]\end{array}$ & EIT $\| 28$ & $3-8 \%$ relative errors & MLP \\
\hline $\begin{array}{l}\text { Wang et al. } \\
\text { (2009) [46] }\end{array}$ & EIT || 32 & $1.64-2.94 \%$ & RBFNN trained by PSO optimization algorithm \\
\hline \multirow{3}{*}{$\begin{array}{l}\text { Lampinen et al. } \\
\text { (1999) [39] }\end{array}$} & \multirow{3}{*}{ EIT } & $\begin{array}{l}6.7 \% \text { classification errors } \\
8.7 \% \text { relative errors in void fraction }\end{array}$ & $\begin{array}{l}\text { Multilayer Perceptron (MLP) neural network } \\
\text { MLP ESC }\end{array}$ \\
\hline & & $\begin{array}{l}5.9 \% \text { classification errors } \\
8.1 \% \text { relative errors in void fraction }\end{array}$ & Bayesian MLP \\
\hline & & $3.4 \%$ relative errors in void fraction & Bayesian MLP, direct void fraction \\
\hline \multirow{3}{*}{$\begin{array}{l}\text { Vehtari and Lampinen } \\
(2000)[47]\end{array}$} & \multirow{3}{*}{ EIT } & $\begin{array}{l}4.7 \% \text { classification errors } \\
16.2 \% \text { errorsin void fraction }\end{array}$ & $\begin{array}{l}\text { MLP ESC } \\
\text { (NNTB3 def) }\end{array}$ \\
\hline & & $\begin{array}{l}4.5 \% \text { classification errors } \\
15.7 \% \text { error in void fraction }\end{array}$ & MLP ESC (decent def) \\
\hline & & $\begin{array}{l}3.8 \% \text { classification errors } \\
6.0 \% \text { error in void fraction }\end{array}$ & Bayesian MLP \\
\hline $\begin{array}{l}\text { Peng and Mo } \\
(2003)[48]\end{array}$ & $\begin{array}{l}\text { EIT \|| } 32 \| \\
\text { neighboring method }\end{array}$ & $\begin{array}{l}\text { 10-4 mean squared } \\
\text { error (MSE) of networks }\end{array}$ & $\begin{array}{l}\text { Multilevel BP neural network } \\
\text { (MBPNN) (three levels) }\end{array}$ \\
\hline $\begin{array}{l}\text { Minhas and Reddy } \\
(2005)[40]\end{array}$ & EIT \| 16 & $8 \%$ limit error & $\begin{array}{l}\text { Four different RBFNNs, corresponding to four } \\
\text { different classifiers, was trained by applying } \\
\text { OLSA }\end{array}$ \\
\hline $\begin{array}{l}\text { Adler and Guardo } \\
(1994)[43]\end{array}$ & EIT $\| 16$ & $0.49-1.38 \%$ & ADALINE network (Adaptive Linear Element) \\
\hline $\begin{array}{l}\text { Ghasemazar and Vahdat } \\
(2007)[49]\end{array}$ & EIT & $1.4 \times 10-5-6.2 \times 10-5 \mathrm{MSE}$ of networks & Several Multilayer Perceptron (MLP) \\
\hline $\begin{array}{l}\text { Miller et al. } \\
(1992)[50]\end{array}$ & EIT & $5 \%$ & Back-projection network \\
\hline $\begin{array}{l}\text { Ratajewicz-Mikolajczak et al. } \\
(1998)[51]\end{array}$ & EIT \| 16 & $3-8 \%$ relative errors & $\begin{array}{l}\text { ANN learning using Cascade Correlation } \\
\text { Learning Algorithm (CASCOR) }\end{array}$ \\
\hline $\begin{array}{l}\text { Takeuchi and Kosugi } \\
\text { (1994) [52] }\end{array}$ & $\begin{array}{l}\text { EIT \| } 18 \| \\
\text { opposite method }\end{array}$ & Error induced by noise appeared about $20 \%$ & $\begin{array}{l}\text { Fletcher-Reeves conjugate gradient method } \\
\text { for BP learning }\end{array}$ \\
\hline \multirow{2}{*}{$\begin{array}{l}\text { Teague et al. } \\
(2001)[53]\end{array}$} & \multirow{2}{*}{ EIT } & $5.6-12 \%$ void fraction errors & $\begin{array}{l}\text { Single-layer feed-forward NN was trained using } \\
\text { gradient descent }\end{array}$ \\
\hline & & $6.15-11.2 \%$ void fraction errors & $\begin{array}{l}\text { Double-layer feed-forward NN was trained using } \\
\text { resilient back-propagation }\end{array}$ \\
\hline
\end{tabular}

\subsubsection{Structure of self-organizing maps}

SOMs consist of elements called 'nodes' or neurons connected topologically, see Figure 7. Each node $i \in N$, with $N$ the dimension of the neural network, is connected to the input vector $j \in M$, with $M$ the dimension of the input vector, and to the vector of weights $w_{i j}$ with $i, j \in N \times M$ and each node has a local influence on their direct neighbours.

Learning takes place iteratively as follows. At each cycle, the distance $d_{i}$ between all weights and the input vector is computed; see eq. 5 . The neuron with the smallest distance is called the winning neuron. Its weights and those of its direct neighbours are modified to reduce the distance to the input vector; see eq. 6 , and their output is computed as the inverse of the distance; see eq. 7. The position of the neurons on a 2-dimensional grid determines the Kohonen map topology.

\subsubsection{The learning algorithm of a SOM}

During the training stage, a distance $d$ (usually an Euclidian or L1 distance) between the input vector $x$ and the neurons' weights $w$ are associated to each output neuron $y$ as in eq. 7:

$$
d_{i}=\sqrt{\sum_{j=1}^{M}\left(x_{j}-w_{i j}\right)^{2}}
$$


where $x_{j}$ is the $j$-th component of the input vector $x$ and $M$ is the dimension of the input vector $x$; $d_{i}$ the distance associated to $i$-th neuron within a population of $N$ neurons. The smaller $d$ is, the closer is the receptive field of the neuron to that input vector.

The output neuron with the smallest distance $d_{i}^{*}=\operatorname{argmin}\left(d_{i}\right)$ is written $i^{*}$ and is then considered as the winner neuron. Its weights are updated following eq. 6 as well as the neurons within a certain neighborhood radius $h_{c i^{*}}[58]$ :

$$
w_{i j}(t+1)=w_{i j}(t)+\varepsilon h_{c i}\left(x_{j}(t)-w_{i j}(t)\right)
$$

where $\varepsilon$ is the learning rate, for iteration time $t$. The Kohonen rule changes the weights of the winner neuron and of its neighbors [59,60], which get closer to the input vector and cause the decreasing of the distance to it. As a result, the Kohonen network learns to classify topologically similar vectors. The output value $y_{i}$ of the neuron $i$ is the inverse of the distance $d_{i}$ measured in eq. 5:

$$
y_{i}=\frac{1}{1+d_{i}}
$$

\section{Imaging reconstruction with Kohonen Neural Networks}

\section{$5.1 \quad$ Experimental setup}

Figure 7 represents a schematic diagram of the overall experimental setup for reconstructing the spatial location of the conductive weights by a Kohonen map from the distribution of the resistance density. A small constant DC current of $200 \mu \mathrm{A}$ is applied to the surface (input vector size of 16 bins), the voltages are measured using the neighboring method of EIT (output vector size of $16 \times 13$ bins) and a Kohonen map of $32 \times 32$ neurons is used to solve the inverse problem of EIT; the electronic hardware is detailed in [36].

The Kohonen network is part of the simulated annealing algorithms which require an exploration/exploitation period to converge. The learning stage is therefore done in two phases, the ordering phase and the tuning phase that control respectively the network plasticity and stability. This is applied here by selecting the number of examples fed to the neurons and the population of neuron to be fed. On the one hand, during the ordering phase, the training set is initialized with a given quantity of the data set and the radius of the neighborhood begins with an initial distance value that decreases to the unit value. This first mechanism allows the weights of neurons to self-organize in the input space consistent with their positions. On the other hand, during the tuning phase, the remaining samples of the data set are used to update the weights of the winner neuron only. This second mechanism allows the weights to change relatively evenly in the input space while maintaining the topology found in the ordering phase.

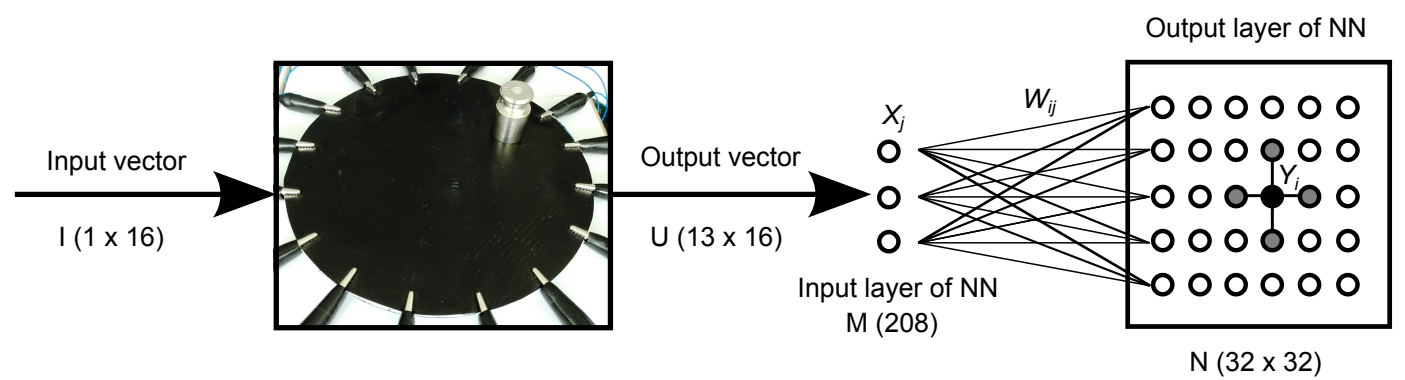

Figure 7. Schematic diagram to estimate the resistivity distribution on the tactile sheet by a Kohonen map based on the EIT method. The input current $I$ is injected at 16 different locations, which give the voltage matrix $U$, the input of the Kohonen map of $32 \times 32$ units. 

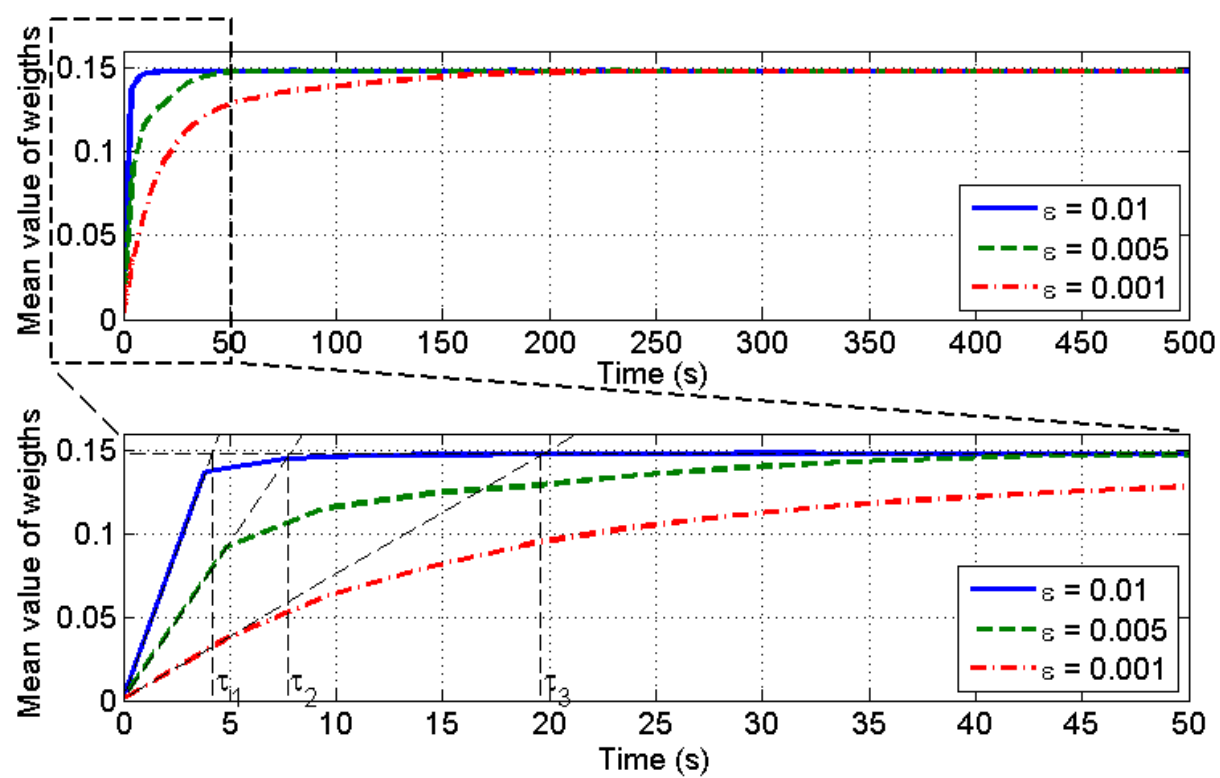

Figure 8. Convergence rate of the Kohonen network during the learning stage based on the mean value of the weights and for different learning steps $\epsilon=0.01,0.005$ and 0.001 . Although the convergence rate is faster for the bigger $\epsilon$, the networks that slowly adapt to the input matrices are also more finely tuned, as it can be seen in the evolution of the entropy value over the weights, which is also a measure of information or complexity within the network.

Simultaneously, we make the learning rate $\varepsilon$ to decrease during the learning period from 0.01 to 0.001 . The neighborhood function $h_{c i}$, a Gaussian function, goes from high values of neighborhood weights (strong local influence) to low values of neighborhood weights (weak local influence). The average values of the weights of the Kohonen Network during the learning period and for different learning rate are depicted in Figure 8.

Figure 8 (top chart) allows us to see properly the evolution of the average weight of the neural network for three $\varepsilon$ values $(0.01,0.005$ and 0.001$)$. Indeed, it appears that the mean value of the weight tends to stabilize at an average weight of 0.1479 . Furthermore, no overshoot of the final value is found. Therefore, the curves obtained can remind the step response of a low-pass filter of the first order. This is why in Figure 8 (bottom chart) the tangents at the origin are drawn leading to construction of time constants $\tau_{1}, \tau_{2}$ and $\tau_{3}$. The results obtained for each time constant are the following: $\tau_{1}=4.2 \mathrm{~s}, \tau_{2}=7.7 \mathrm{~s}$ and $\tau_{3}=19.6 \mathrm{~s}$. In fact, the average network weights stabilizes around a duration of $5 \tau$.

\section{$5.2 \quad$ Experimental Results}

The spatial location of the object and the resistance density distribution were reconstructed with the Kohonen neural network learnt in the previous section. Figure 9 shows the test results of the neural network with $32 \times 32$ neurons in the output layer when an object of $100 \mathrm{~g}$ is moved continuously on the tactile surface. The location of higher neural activity corresponds to the cluster receptive field where the weight is set. The neurons receptive field is large as it englobes many neurons within the field, the cluster moves smoothly and linearly with respect to the spatial displacement of the weight on the tactile sensor. The reconstruction time is about $20 \mathrm{~ms}$ at each iteration.

\subsection{Experiment 2 - EIT vs Neural Networks}

Figure 10 represents the spatial location of the object (weight of $100 \mathrm{~g}$ ) and the resistance density distribution was reconstructed with the MATLAB toolbox EIDORS (Electrical Impedance and 

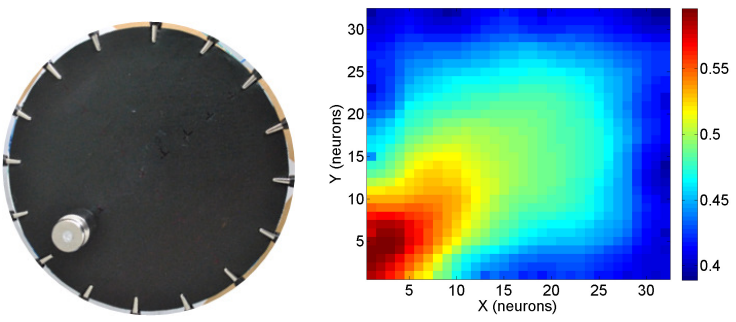

(a) step 1
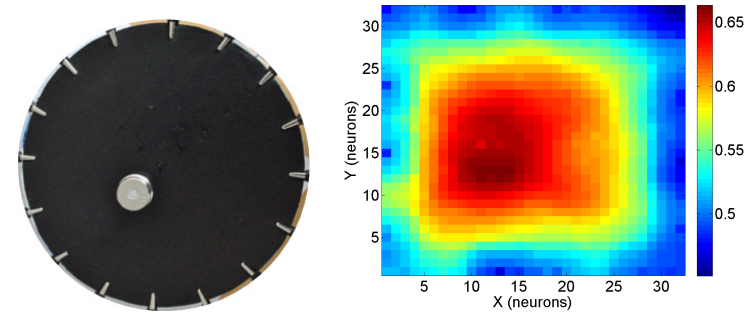

(c) step 3
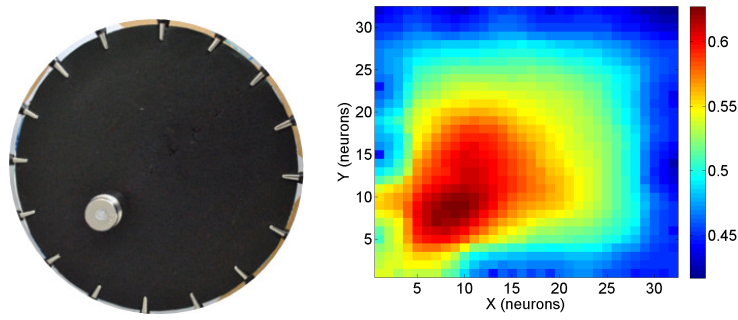

(b) step 2
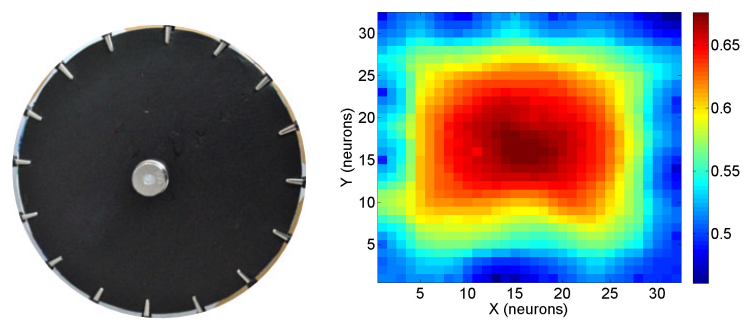

(d) step 4

Figure 9. Position of the object (weight $=100 \mathrm{~g}$ ) on the conductive material and the SOM reconstruction (network size 32 $\times 32$ neurons)

Diffused Optical Reconstruction Software) [61]. This software implements three tools for the mesh generation, the forward problem and the inverse problem [62]. The reconstruction time with EIDORS takes $260 \mathrm{~ms}$ to complete each matrix of tension measurements, which is ten times longer than for the SOM method (20ms).
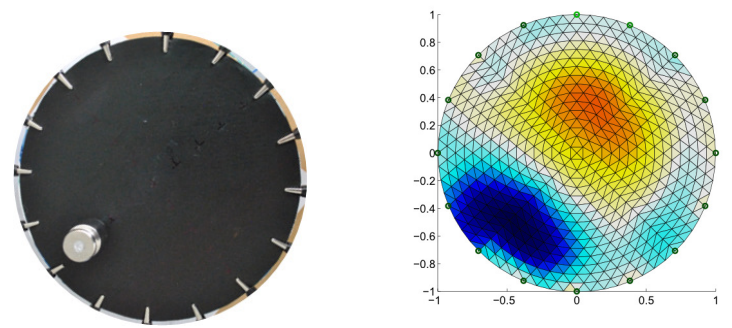

(a) step 1
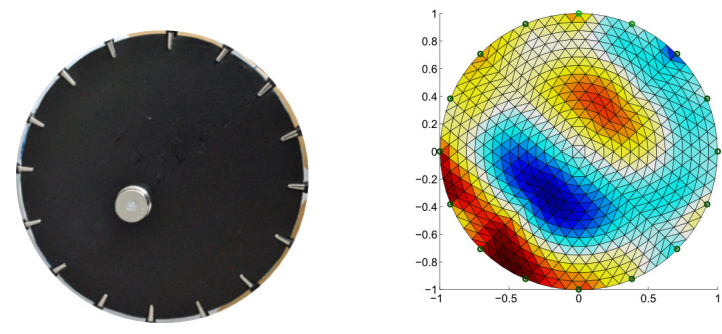

(c) step 3
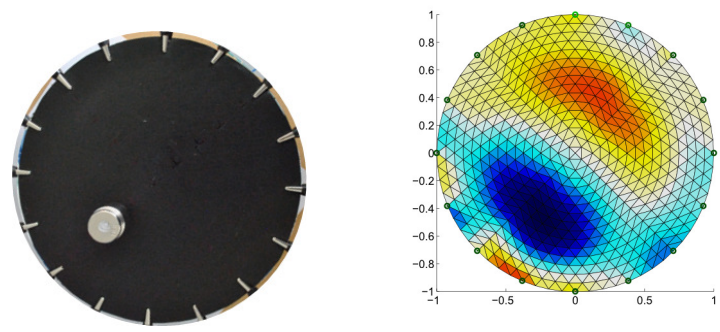

(b) step 2
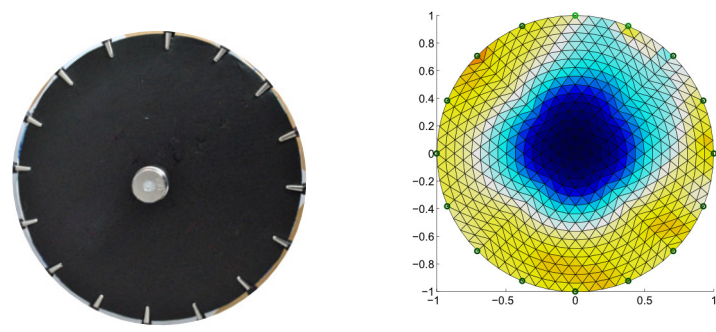

(d) step 4

Figure 10. 2 dimensional image reconstruction using EIDORS of a FEM model with 1024 elements. The hyper parameter $\alpha$ (regularization weight) is set to $10^{-3}$. Blue and reds areas are the areas with higher and lower resistivity respectively.

Initially, we have estimated the no-load resistance distribution of the conductive fabric and we have used it as a reference for calibration. This approach allows to measure the relative change in the resistance distribution compared to the reference state and it is known as difference imaging or dynamic imaging [34]. It consists on the estimation of time-varying changes in resistance 


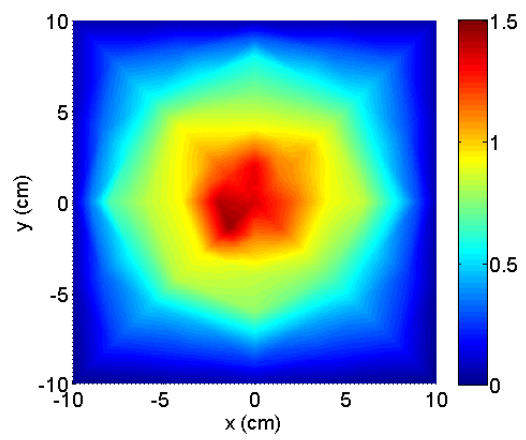

(a) EIDORS

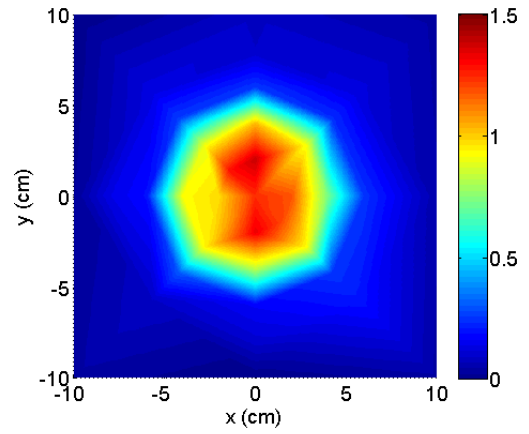

(b) Kohonen map

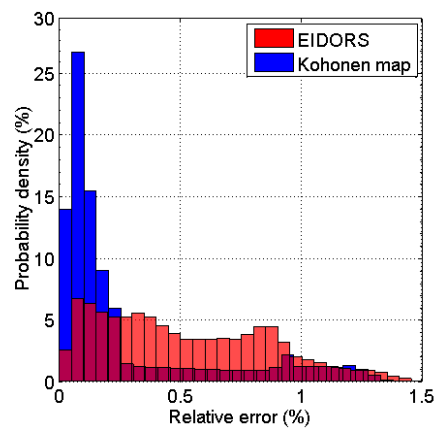

(c) Probability density

Figure 11. Relative error (in \%) of the determination of the position of the object on the conductive material: (a) classical approach reconstruction (Matlab toolbox EIDORS); (b) neural network image reconstruction (Kohonen Map 32 x 32 neurons). (c) probability density of the relative error on the surface of the tactile sensor.

between 2 particular points. If we suppose that the variation in the impedance is low, a linearized algorithm can be used to solve the problem in only one step, which will be close to real-time operations. Thus, there is no iterative computation involved and the real resistance distribution does not need to be computed. This type of image reconstruction in EIT is considered more robust to noise and to electrode positioning errors compared to static imaging.

We present in Figure 11 (a) and (b) the relative position error of an object (100g) on the conductive material for the classical reconstruction approach (Matlab toolbox EIDORS) and for the neural network image reconstruction approach (the Kohonen Map). Other metric measures were proposed in [25], such as 'point localization'.

One can see that the relative error does not exceed $1.5 \%$ for these two approaches and the largest error occurs at the center of the conductive material for both.This is due to the decreased sensitivity of the skin as the function of the distance from the electrodes. Along the edges of the conductive fabric of the Kohonen network can determine the position of the object with a greater accuracy by the classical method (within the area of up to $5 \mathrm{~cm}$ from the edges the error is below $0.5 \%)$. On the edges the error decreases to less than $1 \mathrm{~mm}$. When the Kohonen network increases in size $(13 \times 16,32 \times 32,64 \times 64)$, the maximum relative error is reduced. Nevertheless, the maximum localization error on the artificial skin is less than $1.5 \%$ at the center position, which means that the software EIDORS and NN have a high accuracy with approximately a distance error of $7 \mathrm{~mm}$. Analysis of the error probability density (see Figure 11 (c)) shows that the classical method using EIDORS produces an relative error that is always distributed uniformly, whereas the proposed method with Kohonen map produces more common errors for values less than $0.25 \%$ and less in range up to $1.5 \%$.

\subsection{Experiment 3 with neural networks}

\subsection{1 touch and multitouch}

In this experiment, we investigate the ability of our neural architecture to discriminate the spatial location of multiple contact points, up to three with various weights, see Figure 12. Our circular tactile sensor is represented again with a Kohonen network of 1024 neurons (32 by 32 neurons). We display in Figure 12 (a) the experimental setup for a weight of $100 \mathrm{~g}$ with the corresponding neural activity of the Kohonen map where the location zone can be clearly identified by the high activity level above 0.8. The neural map activity for two weights held on the tactile surface is shown in Figure 12 (b) for which two clusters are found. The activity level of the second cluster, which corresponds to the second object added, is slightly lower than the activity of the first one as it has a smaller weight of $50 \mathrm{~g}$. The overall neural activity is also reduced as having two objects instead of one diminishes the voltage value. The activity values 

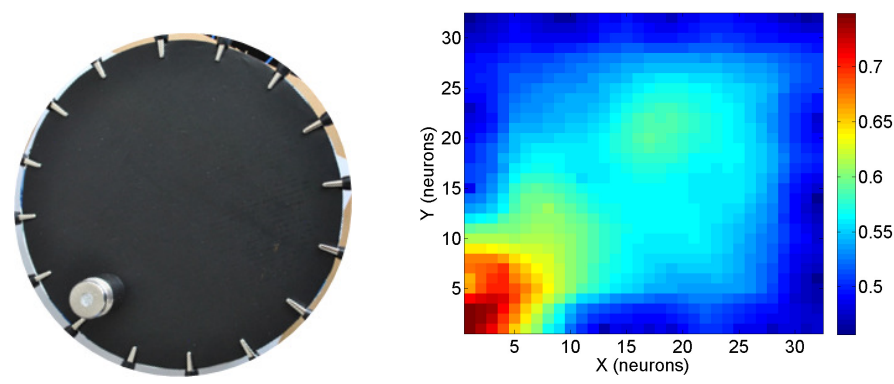

(a) Single contact point (weigth of $100 \mathrm{~g}$ ).
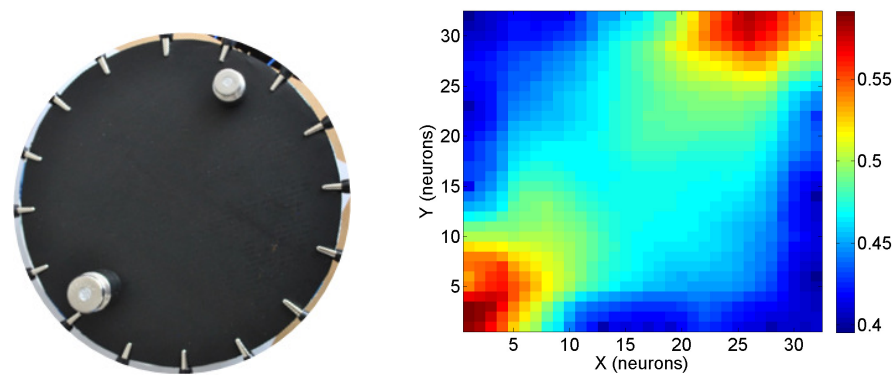

(b) Two contact points (weigths of $100 \mathrm{~g}$ and $50 \mathrm{~g}$ ).
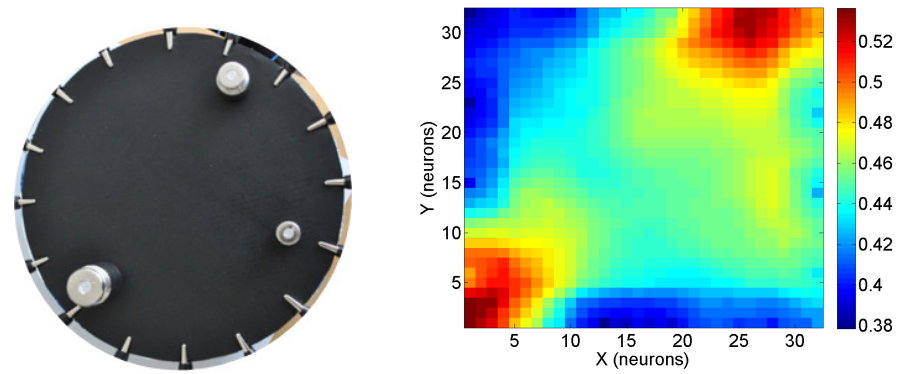

(c) Three contact points (weigths of $100 \mathrm{~g}, 50 \mathrm{~g}$ and $20 \mathrm{~g}$ ).

Figure 12. Neural activity of the Kohonen map for multi-touch task for one, two or three weights of $100 \mathrm{~g}, 50 \mathrm{~g}$ and $20 \mathrm{~g}$; resp (a), (b) and (c). The neurons are able to estimate correctly and simultaneously objects of different location and weights.

of neural clusters are around 0.6 in the two areas. Finally, we add a third object of $20 \mathrm{~g}$ on the surface and the Kohonen network is capable to identify its spatial location with a third cluster of lower intensity with respect to the others of about 0.48; see Figure 12 (c). Although any new object held on the surface increases the total pressure on it and decreases proportionally the sensor's impedance, the neural network is able to adapt to this change and to be robust to multi-touch. Thus, for the first object with a constant weight of $100 \mathrm{~g}$, its location is computed for a neural activity up to 0.8 , then for the second object up to 0.6 and for the third object, up to 0.55 .

\subsection{2 with different material shapes}

We verified in the previous experiment that the Kohonen map self-organized effectively to preserve the topology of the input structure for the spatial location of objects on the conductive surface. This is another advantage of the Kohonen map over parametric methods, which can adapt dynamically to the topology of the incoming structure. The spatial location of a contact point cannot be determined using the parametric methods if the shape of the tactile surface is unknown or modified, the same is true for the neural networks that do not have any topology like RBF or multi-layer perceptrons. 


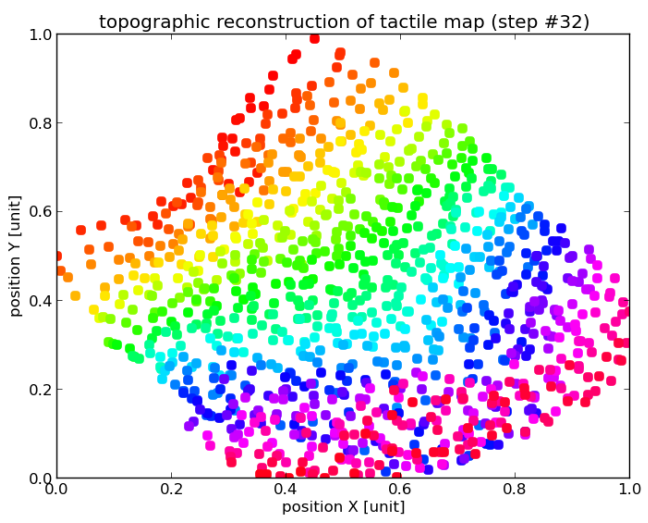

(a) Round-shaped surface

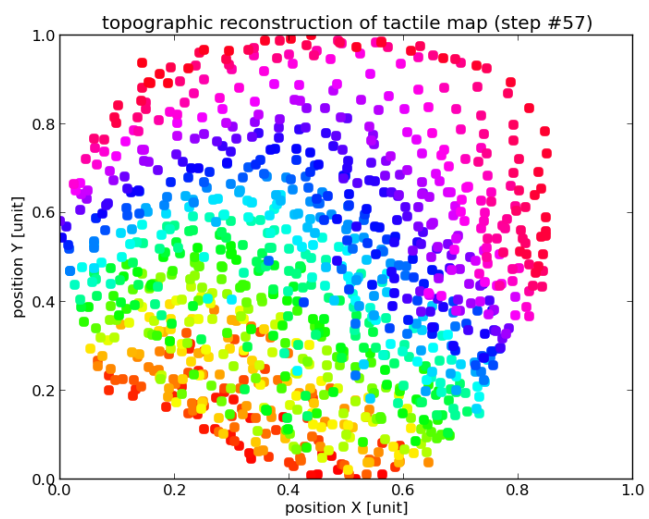

(b) Square-shaped surface

Figure 13. Topological reconstruction from the Kohonen neurons' synaptic weights using the graphical display based on the Fruchterman-Reingold algorithm for two different type of conductive surface, a round in (a) and a square in (b).

Figure 13 shows the topology of two reconstructed Kohonen map using the FruchtermanReingold layout algorithm [63] from a square tactile surface and a round tactile surface, respectively (a) and (b). This algorithm is a visualization technique used in graph theory and in neural networks for analyzing a network's topology. Although very caricatural, the FR algorithm has been used for molecular placement simulations and can serve here to determine the topology of the Kohonen map. It is based on the physical modeling of a springs-masses network with repulsive and attractive forces. The forces are computed from a distance between the neurons weights, for which neighboring neurons with similar weights see their dynamics tightened by attractive forces. We use the jet color layout discretized to 1024 colors associated with each neuron of the Kohonen maps with respect to their index.

As one can see from the graphs, the nodes attempt to reconstruct the topology of the tactile surface with a round-like shape in (a) and a square-like map in (b), respecting the nonlinearities of the corners.

\subsection{3 with different objects}

One extension from detecting multiple contact points on the tactile surface is to identify spatially ordered contact points as it is in the visual pattern recognition of objects. Four archetypal shapes are used to test the effectiveness of the SOM to perceive the actual stimulated profile from the neural activity on the active sensor area: a triangular prism (three sharp angles), a cube (four blunt angles), a ruler (two blunt angles) and a cylinder. Figure 14 shows the activity of the Kohonen neurons for four different shapes with the topology found by the Fruchterman-Reingold layout algorithm in Figure 13 (a), red color reflects the neuron activity above a certain threshold (the same for the four shapes).

As one can see, it is possible to discriminate to some extent the object shape from the neural activity, although the spatial resolution is not fully respected and the topology slightly distorted. The sharp angles of objects permit to discriminate each object more easily and to find their orientation. These properties - to discriminate objects and find their orientation are not trivial as the Kohonen map has learnt from individual tactile points; for instance, it is not clear if it is possible to reduce the shape of objects to a linear combination of individual points: we do not know whether or not the electrical field of one complex shape object can be easily reduced to the electrical field of multiple points. This method shows therefore its limits and the need to acquire other types of signals (visual, proprioceptive or other mechanoreceptors) to discriminate better object shapes. 

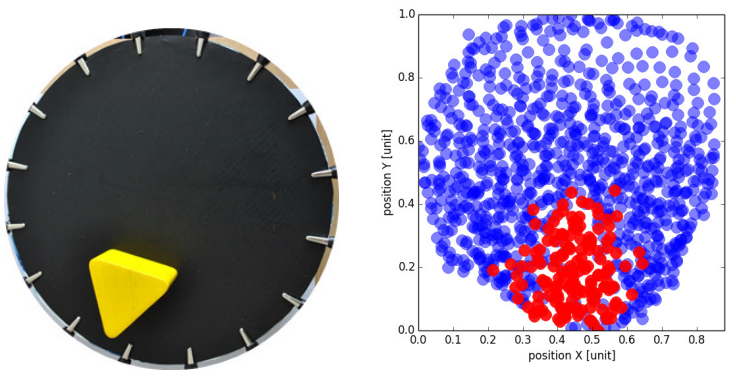

(a) a prism

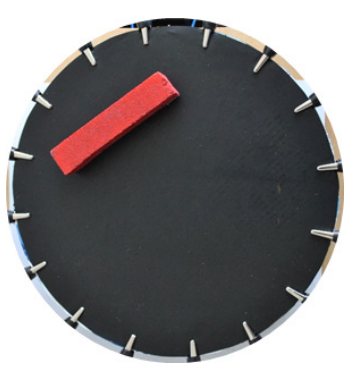

(c) a parallelipedic shape

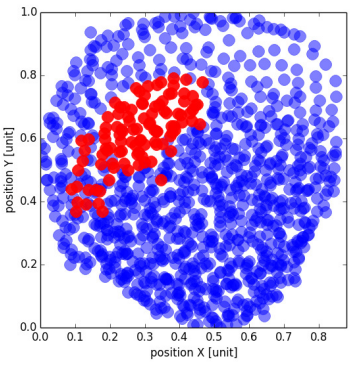

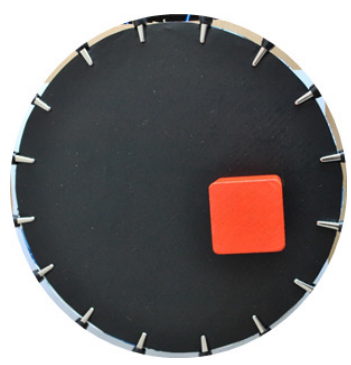

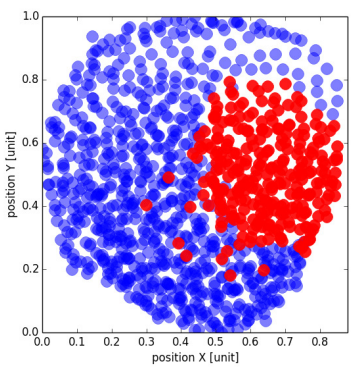

(b) a cube
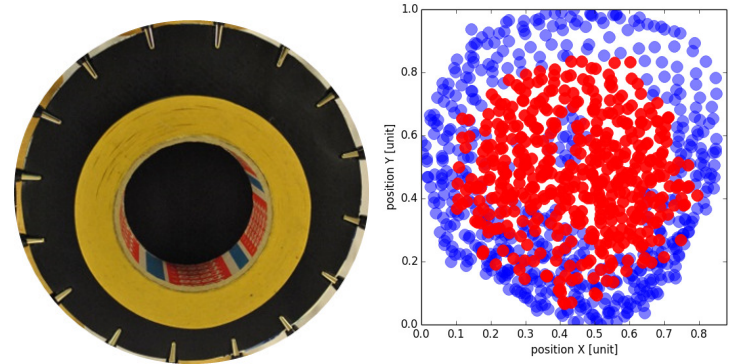

(d) an unfilled cylinder

Figure 14. Activity-level of the Kohonen neurons in presence of various shape and above a certain threshold.

\section{Conclusion}

The sense of touch is an important feature to provide in robots in order to represent their body and to interact with the environment. It requires to discover the configuration of their sensors and to learn the structure of sensorimotor information. Moreover, this feature should be adaptive so that changes can be overcome as well: (i) in the sensor configuration, (ii) in the number of contact points, (iii) in the pressure range or (iv) with the presence of noise.

In this paper, we present a bio-inspired solution for reconstructing the topology of a tactile device and for sensing the external contact points on it. Neural networks can permit to avoid for the experimenter to identify himself the sensor properties and to configure the important parameters of the reconstruction method that can be different from the datasheet specification and during its use due to the material deterioration and damage. In comparison to classical methods, neural networks can generalize from a large range of sensory inputs off-the-shelf without an a priori on the sensor structure. Our motivation is to develop in the long run a whole-body artificial skin for humanoid robots with features emulating the human tactile perception such as providing the spatial location of the physical contact point within the robot coordinate frame. To our knowledge, we are the first to use neural networks for EIT image reconstruction of tactile devices in the robotic domain and to use unsupervised techniques for EIT image reconstruction in the literature. We did not compare our approach with other ANNs because advantages and drawbacks of each family of ANN are already well documented [64].

The Kohonen network that we used has the advantages to model the topographic organization in the somatopic area in the cortex similar to what is done in [57]. Its functioning is based on a metric distance between the input vector and the neuron's weights, which assumes that the sensory inputs do have a topology and that a tiny displacement of a contact point on the material surface will have an effect on the neurons' activity and will be translated proportionally in term of a neural distance to each neuron's receptive field. This characteristic endows the Kohonen map to be tolerant to noise and to emulate well multi-touch contact points and objects of different shapes. Moreover, the Kohonen map showed advantages over the classical reconstruction methods in terms of performance (good resolution accuracy) with the possibility to sense tactile 
inputs in real-time (in average $20 \mathrm{~ms}$ versus $262 \mathrm{~ms}$ for EIDORS using the difference imaging of EIT). Although it has been reported that the difference EIT image reconstruction technique can be very fast and can achieve $40-50 \mathrm{~Hz}$ [65], the computational cost or complexity of EIT reconstruction techniques has been shown to be a drawback when the matrix size augments [66]. The computational complexity of EIT methods requires to inverse one matrix of dimension $n$, which costs computationally at least $O\left(n^{2} \times m\right)$, where $m$ is the number of finite elements of the FEM, which means that as the matrix augments -, the size of the tactile sheet and the number of electrodes,- the computational time for image reconstruction will increase asymptotically. Instead, the computational cost of neural networks of $m$ neurons and for the same input matrix of dimension $n$ is $O(n \times m)$, because the network output depends on the computation of all the neurons within the neural network. In neural networks, the dimension $m$ is often chosen inferior to $n$, for generalization purpose but in our tests, we chose $m=1024$ for both algorithms in order to compare them. This means that the computational time for output approximation is increasing linearly with the size of the neural network. Furthermore, we suggest that the computation done by the $\mathrm{NN}$ is similar to the filtering process known as super-resolution in image processing to enhance the spatial resolution and used recently with tactile devices [19] using Bayesian techniques. NN interpolate several EIT samples acquired during the learning period to enhance the spatial resolution of the tactile device.

Nonetheless, SOMs are part of the model-free and unsupervised techniques, which are known to be less efficient than the supervised learning methods because the model of the desired state is given in advance and exploited to minimize the error. Thus, we expect that multi-layer perceptrons, deep networks, radial basis function, or support-vector machines to name a few will give better performances in terms of convergence rate and accuracy than the Kohonen map that we have employed. For these reasons, we believe that supervised neural networks can provide in the future interesting and efficient solutions in terms of speed and error approximation to the EIT image reconstruction problem.

SOMs have instead as advantages to learn online the topology of the input space (the geometry of the tactile sheet) without any a priori knowledge. Furthermore, they are easy to interpret and more plausible biologically. For instance, each neuron of the SOM map models a mechanoreceptor (e.g., the low-pass filter Merkel type) and each neuron learns its own position in the tactile sheet as well as its own spatial resolution. The precision acquired depends on the learning (or developmental) stage done, which is not the case for unit-based devices that have only a static and uniform spatial distribution. ctually, we are working on the modeling of different types of mechanoreceptors using our method.

Moreover, and as similar to the experience done in section 4.5.3 with the Kohonen map, we did the blind-test to recognize the shape of objects on the surface of one arm's skin and we could not easily discriminate the different shapes by just pressing the object on it. This is in accordance with research studies showing that the other senses -vision, proprioception, and audition- and the combination of the mechano-receptors altogether contribute to tactile perception $[67,68]$. Therefore, the aim of our future research will be to investigate the integration of other senses like vision or proprioception towards the perception of ones own body and modeling of the sense of ownership in a humanoid robot based on the tactile sense [69, 70].

\section{Acknowledgements}

The authors would like to acknowledge Artem Melnyk for discussions, Agence Universitaire de la Francophonie (AUF) and Academie Francophone des Savoirs (University of Cergy-Pontoise) for research grant support, ENSEA and CNRS chaire d'excellence for funding grant. 


\section{References}

[1] Tabot G, Kim S, Winberry J, Bensmaia S. Restoring tactile and proprioceptive sensation through a brain interface. Neurobiol Dis. 2014;

[2] Buonomano D, Merzenich M. Cortical plasticity: from synapses to maps. Annual Review of Neuroscience. 1998;21:149-186.

[3] Kaas J, Qi H. The reorganization of the motor system in primates after the loss of a limb. Restorative neurology and neuroscience. 2004;22(3-5):145-152.

[4] Hoshi T, Shinoda H. Robot skin based on touch-area-sensitive tactile element. IEEE International Conference on Robotics and Automation, ICRA. 2006;:3463-3468.

[5] Roncone A, Hoffmann M, Pattacini U, Metta G. Automatic kinematic chain calibration using artificial skin: self-touch in the icub humanoid robot. IEEE International Conference on Robotics and Automation, ICRA. 2014;:2305-2312.

[6] Cadoret G, Smith A. Friction, not texture, dictates grip forces during object manipulation. Journal of Neurophsiology. 1996;75(5):1963-1969.

[7] Flanagan J, Wing A. Modulation of grip force with load force during point-to-point arm movements. Experimental Brain Research. 1993;95(1):131-143.

[8] Johansson R, Westling G. Roles of glabrous skin receptors and sensorimotor memory in automatic control of precision grip when lifting rougher or more slippery objects. Experimental Brain Research. $1984 ; 56(3): 550-564$.

[9] Duchaine V, Lauzier N, Baril M, Lacasse M, Gosselin C. A flexible robot skin for safe physical human robot interaction. IEEE International Conference on Robotics and Automation, ICRA. 2009; :3676-3681.

[10] Bongard J, Zykov V, Lipson H. Resilient machines through continuous self-modeling. Science. 2006; 314(5802):1118-1121.

[11] Dahiya R, Metta G, Valle M, Sandini G. Tactile sensingfrom humans to humanoids. IEEE Transactions on Robotics. 2010;26(1):1-20.

[12] Lee M, Nicholls H. Tactile sensing for mechatronicsa state of the art survey. Mechatronics. 1999; $9(1): 1-31$.

[13] Tiwana M, Redmond S, Lovell N. A review of tactile sensing technologies with applications in biomedical engineering. Sensors and Actuators A: Physical. 2012;179:17-31.

[14] Lee HK, Chang SI, Yoon E. A flexible polymer tactile sensor: fabrication and modular expandability for large area deployment. Journal of Microelectromechanical Systems,. 2006;15(6):1681-1686.

[15] Meister E, Zilberman I, Levi P. Fuzzy logic based sensor skin for robotic applications. International Conference on Artificial Intelligence, ICAI. 2012;2:3-9.

[16] Cannata G, Maggiali M, Metta G, Sandini G. An embedded artificial skin for humanoid robots. IEEE International Conference on Multisensor Fusion and Integration for Intelligent Systems, MFI. 2008;:434-438.

[17] Cheng MY, Chang WY, Tsao LC, Yang SA, Yang YJ, Shih WP, Chang FY, Chang SH, Fan KC. Design and fabrication of an artificial skin using pi-copper films. IEEE 20th International Conference on Micro Electro Mechanical Systems, MEMS. 2007;:389 - 392.

[18] Pfeifer R, Iida F, Lungarella M. Self-organization, embodiment, and biologically inspired robotics. Science. 2007;318(5853):1088-1093.

[19] Lepora N, Martinez-Hernandez U, Evans M, Natale L, Metta G, Prescott T. Tactile superresolution and biomimetic hyperacuity. IEEE Robotics and Automation Society. 2005;31(3):605-618.

[20] Patel G, Kaplan D, Snyder L. Topographic organization in the brain: searching for general principles. Trends in Cognitive Sciences. 2014;18(7):351-363.

[21] Schmeder A, Freed A. Support vector machine learning for gesture signal estimation with a piezo resistive fabric touch surface. In: Beilharz K, Bongers B, Johnston A, Ferguson S, editors. Proceedings of the international conference on new interfaces for musical expression. Sydney, Australia. 2010. p. 244-249.

[22] Johnsson M, Balkenius C. Sense of touch in robots with self-organizing maps. IEEE Transactions on Robotics. 2011;27(3):498.

[23] Ratnasingam S, McGinnity T. Object recognition based on tactile form perception. IEEE Workshop on Robotic Intelligence In Informationally Structured Space (RiiSS). 2011;:26-31.

[24] Webster J. Medical instrumentation application and design. third edition ed. New York: Wiley. 2007.

[25] Adler A, Arnold J, Bayford R, Borsic A, B B, Dixon P, Faes T, Frerichs I, Gagnon H, Garber Y, 
Grychtol B, Hahn G, Lionheart W, Malik A, Patterson R, Stocks J, Tizzard A, Weiler N, Wolf G. Greit: a unified approach to 2d linear eit reconstruction of lung images. Physiol Meas. 2009; 30:S35-S55.

[26] Kato Y, Mukai T, Hayakawa T, Shibata T. Tactile sensor without wire and sensing element in the tactile region based on eit method. IEEE Sensors. 2007;:792-795.

[27] Yao A, Soleimani M. A pressure mapping imaging device based on electrical impedance tomography of conductive fabrics. Sensor Review. 2012;32(4):310- 317.

[28] Nagakubo A, Alirezaei H, Kuniyoshi Y. A deformable and deformation sensitive tactile distribution sensor. IEEE International Conference on Robotics and Biomimetics, ROBIO. 2007;:1301-1308.

[29] Alirezaei H, Nagakubo A, Kuniyoshi Y. A highly stretchable tactile distribution sensor for smooth surfaced humanoids. 7th IEEE-RAS International Conference on Humanoid Robots. 2007;:167-173.

[30] Alirezaei H, Nagakubo A, Kuniyoshi Y. A tactile distribution sensor which enables stable measurement under high and dynamic stretch. IEEE Symposium on 3D User Interfaces (3DUI ). 2009;:87 93.

[31] Tawil D, Rye D, Velonaki M. Improved image reconstruction for an eit-based sensitive skin with multiple internal electrodes. IEEE Transactions on Robotics. 2011;27(3):425 - 435.

[32] Brown B, Seagar A. The sheffield data collection system. Clinical Physics and Physiological Measurement, volume =. 1987;

[33] Holder D, editor. Electrical impedance tomography: Methods, history and applications,. UK: Institute of Physics Publishing. 2005.

[34] Adler A, R G. Electrical impedance tomography: Regularized imaging and contrast detection. IEEE Transactions on Medical Imaging. 1996;15(2):170-179.

[35] Vauhkonen M, Vadasz D, Karjalainen E PA amd Somersalo, , Kaipio J. Tikhonov regularization and prior information in electrical impedance tomography. IEEE Transactions on Medical Imaging. 1998;17(42):285- 293.

[36] Pugach G, Khomenko V, Melnyk A, Pitti A, Henaff P, Gaussier P. Electronic hardware design of a low cost tactile sensor device for physical human-robot interactions. IEEE XXXIII International Scientific Conference Electronics and Nanotechnology, ELNANO. 2013;:445-449.

[37] Heikkinen L, Vauhkonen M, Savolainen T, Kaipio J. Modelling of internal structures and electrodes in electrical process tomography. Measurement Science and Technology. 2001;12(8):1012 - 1019.

[38] Tarvainen M, Vauhkonen M, Savolainen T, , Kaipio J. Boundary element method and internal electrodes in electrical impedance tomography. International Journal for Numerical Methods in Engineering. 2001;50(4):809-824.

[39] Lampinen J, Vehtari A, Leinonen K. Application of bayesian neural network in electrical impedance tomography. IEEE International Joint Conference on Neural Networks (IJCNN '99). 1999;6:39423947.

[40] Minhas AS, Reddy MR. Neural network based approach for anomaly detection in the lungs region by electrical impedance tomography. Physiological measurement. 2005;26(4):489-502.

[41] Wang C, Lang J, Wang HX. Rbf neural network image reconstruction for electrical impedance tomography. IEEE Proceedings of 2004 International Conference on Machine Learning and Cybernetics. 2004;4:2549-2552.

[42] Pandey V, Clausen B. Intelligent systems based anomaly detection in thoracic region by electrical impedance tomography technique. Ganpat University Journal of Engineering and Technology. 2011; $1(1): 1-3$.

[43] Adler A, Guardo R. A neural network image reconstruction technique for electrical impedance tomography. IEEE Transactions on Medical Imaging. 1994;13(4):594-600.

[44] Nejatali A, Ciric I. Impedance image reconstruction using neural networks. IEEE Antennas and Propagation Society International Symposium - APSURSI. 1997;3:1726 - 1729.

[45] Stasiaka M, Sikorab J, Filipowiczb S, Nitab K. Principal component analysis and artificial neural network approach to electrical impedance tomography problems approximated by multi-region boundary element method. Engineering Analysis with Boundary Elements. 2007;31(8):713 - 739 .

[46] Wang P, Xie L, Sun Y. Application of pso algorithm and rbf neural network in electrical impedance tomography. IEEE 9th International Conference on Electronic Measurement and Instruments, ICEMI. $2009 ;: 2-517-2-521$.

[47] Vehtari A, Lampinen J. Bayesian mlp neural networks for image analysis. 11th Scandinavian Conference on Image. 2000;21(13-14):1183 - 1191.

[48] Peng Y, Mo YL. Locating impedance change in electrical impedance tomography based on multilevel 
bp neural network. Journal of Shanghai University (English Edition). 2003;7(3):251 - 255.

[49] Ghasemazar M, Vahdat B. Error study of eit inverse problem solution using neural networks. IEEE International Symposium on Signal Processing and Information Technology. 2007;:894 - 899.

[50] Miller A, Blott B, Hames T. Neural networks for electrical impedance tomography image characterisation. Clinical Physics and Physiological Measurement. 1992;13(Supplement A):119 - 23.

[51] Ratajewicz-Mikolajczak, Shirkoohi G, Sikora J. Two ann reconstruction methods for electrical impedance tomography. IEEE Transactions on Magnetics. 1998;34(5):2964 - 2967.

[52] Takeuchi J, Kosugi Y. Neural network representation of finite element method. Neural Networks. 1994;7(2):389 - 395.

[53] Teague G, Tapson J, Smit Q. Neural network reconstruction for tomography of a gravel-air-seawater mixture. Measurement Science and Technology. 2001;12(8):1102 - 1108.

[54] Kohonen T. Self-organized formation of topologically correct feature maps. Biological Cybernetics. 1982;43:59-69.

[55] Kohonen T. Self-organizing maps. third extended edition ed. Vol. 30 of Springer Series in Information Sciences. New York: Springer. 2001.

[56] Egmont-Petersen M, Ridder D, Handels H. Image processing with neural networks - a review. Pattern Recognition. 2002;35(10):2279-2301.

[57] Detorakis G, Rougier N. A neural field model of the somatosensory cortex: Formation, maintenance and reorganization of ordered topographic maps. PLoS ONE, Public Library of Science. 2012; $7(7): \mathrm{e} 40257$.

[58] Beale M, Hagan M, Demuth H. Neural network toolbox 7.0.3: Users guide. Natick, USA: The MathWorks, Inc.. 2012.

[59] Gallistel C. The organization of learning. MIT Press, Cambridge, MA. 1993.

[60] Lieberman D. Learning: Behavior and cognition. 2nd ed. Pacific Grove, CA. 1993.

[61] Adler A, Lionheart W. Uses and abuses of eidors: an extensible software base for eit. Physiological Measurement. 2006;27(5):25 - 42 .

[62] Vauhkonen M, Lionheart W, Heikkinen L, Vauhkonen P, Kaipio JP. A matlab package for the eidors project to reconstruct two-dimensional eit images. Physiological Measurement. 2001;22(1):107 - 111.

[63] Fruchterman T, Reingold E. Graph drawing by force-directed placement. Software: Practice and Experience. 1991;21:1129-1164.

[64] Haykin S. Neural networks: A comprehensive foundation. New York: Macmillan. 1994. Available from: http://books.google.fr/books?id=PSAPAQAAMAAJ.

[65] Silvera-Tawil D, Rye D, Soleimani M, Velonaki M. Electrical impedance tomography for artificialsensitive robotic skin: A review. IEEE Sensors Journal. 2015;15(4):1129-1164.

[66] Boyle A, Borsic A, Adler A. Addressing the computational cost of large eit solutions. Physiol Meas. 2012;33(5):787-800.

[67] Gallace A, Spence C. Touch and the body: The role of the somatosensory cortex in tactile awareness. Psyche. 2007;16(1):31-67.

[68] Gallace A, Spence C. The cognitive and neural correlates of 'tactile consciousness': A multisensory perspective. Consciousness and Cognition. 2008;17:370-407.

[69] Pitti A, Alirezaei H, Kuniyoshi Y. Cross-modal and scale-free action representations through enaction. Neural Networks. 2009;22:144-154.

[70] Pitti A, Kuniyoshi Y, Quoy M, Gaussier P. Modeling the minimal newborn's intersubjective mind: The visuotopic-somatotopic alignment hypothesis in the superior colliculus. PLoS ONE. 2013; 8(7):e69474. 